Journal of Engineering and Applied Sciences 14 (24): 9557-9561, 2019

ISSN: 1816-949X

(C) Medwell Journals, 2019

\title{
Establishing Reasonable Safety Management Costs to Ensure Event Safety
}

\author{
${ }^{1}$ Kyung Hwan Ko, ${ }^{2} \mathrm{Jin}$ Seop Lee, ${ }^{3}$ Wang Gi Lee and ${ }^{2} \mathrm{Ki}$ Sang Son \\ ${ }^{1} \mathrm{CJ}$ ENM, Seoul, Korea \\ ${ }^{2}$ Department of Safety Engineering, Seoul National University of Technology, Seoul, Korea \\ ${ }^{3}$ Medical Center, Kyung Hee University, Seoul, Korea
}

\begin{abstract}
In a theater venue that hosts about 40 million people a year, safety accidents are a non-negligible risk. Accidents can be very dangerous when there is an unrestricted crowd in a limited place. In 2006, dozens of people were crushed to death in the Sangju outdoor concert hall. As a result, the National Emergency Management Agency produced a safety management manual for the venue in 2007 . The manual intended to lay out safety management rules for performances and events administered by local governments throughout the country. However, the guidelines were not implemented as they were not turned into legislation.
\end{abstract}

$\underline{\text { Key words: Safety management, safety costs, event safety, non-negligible risk, legislation, concert hall }}$

\section{INTRODUCTION}

In a theater venue that hosts about 40 million people a year, safety accidents are a non-negligible risk. Accidents can be very dangerous when there is an unrestricted crowd in a limited place. In 2006, dozens of people were crushed to death in the Sangju outdoor concert hall. As a result, the National Emergency Management Agency produced a safety management manual for the venue in 2007. The manual intended to lay out safety management rules for performances and events administered by local governments throughout the country. However, the guidelines were not implemented as they were not turned into legislation.

Despite the occurrence of both big and small venue accidents, the Ministry of Employment and Labor's "Occupational Accident Occurrence in 2016" report only listed them as a general type of industrial accident in the "entertainment, culture and sports-related business" category. Accidents at venues remain a blind spot in domestic safety.

The causes of these accidents can be classified as follows: 21 cases related to design, 10 cases to an insufficient safety plan, 2 cases to insufficient funding for education, 11 cases to inexperienced operation, 13 cases of communication failure, 15 cases of negligence, 13 cases related to aging, 1 case of arson, 10 cases of carelessness and 4 others.

In the past, performance venue managers were required to establish a disaster countermeasure plan (safety management plan) setting out prevention measures for fire and casualties and to communicate it to the local government head.
These preventive plans for disasters were not effective enough as there was no obligation to revise them after reporting them once at the time of registration. However, a more recent law now requires venue managers to submit an updated disaster countermeasure plan every year (Kim, 2015; Park, 2014; Choi, 2016).

When the submitted plan is considered insufficient, local governments have laid out grounds for requesting additional measures to improve the safety management at the venue. Moreover, it is necessary to examine how effective it is to set appropriate standards for the investment costs of facility safety management which are considered by the performance venue owners as expenses (Steele, 1996; Ko, 2018).

This study surveyed members of the audience, staffs and hosts and analyzed their answers using frequency, variance and correlation analyses. A regression analysis was conducted to find the proper rate of safety management expenses in theaters, to recognize the problems in domestic theaters and to suggest improvement plans.

\section{MATERIALS AND METHODS}

Devising survey questionnaires: In this study, we surveyed about 100 people (mainly viewers, staffs and performers) who were likely to recognize risks first during a performance or an event. We then also asked 15 performance safety experts to answer a questionnaire.

The first questionnaire survey was designed to identify problems with the application of performance laws in terms of risk assessment and industrial

Corresponding Author: Kyung Hwan Ko, CJ ENM, Seoul, Korea 
safety health law as well as problems with general performance events, operation plans and the revised performance laws.

The results of the first questionnaire were analyzed with frequency and correlation analyses using the SPSS statistical program to identify the risks involved in performance safety management.

A survey on the use of safety management expenses according to the type of performance venue was then conducted to investigate the safety management expenses applied to limited indoor spaces and outdoor spaces and according to the size of the audience. The findings were used to investigate the way expenses were allocated to venues and to suggest improvements.

\section{Distribution of survey questionnaires}

Inter-tier surveys and methods: The safety management reality and degrees of awareness of three classification tiers were investigated for organizers, sponsors and audience members in the performance industry. We investigated the level of safety achieved-starting from stage performance planning and the level of investment and interest in safety management applied to the operation of venues.

Survey method for organizers, sponsors and audience: We surveyed the overall safety mindset related to domestic performance and whether the guidelines for safety organization, safety management costs, safety education, etc., forming part of the disaster response plan stipulated in the recently revised performance law were being realistically implemented. Moreover, the applicability of the industrial safety and health law and the operation of risk assessment were examined.

Devising regression equations: There is a need to know how safety expenses are applied and how their application can be improved. Therefore, we conducted a survey on the use of safety management expenses according to the type of performance to determine the safety management fees and cost items for limited indoor spaces, outdoor spaces and different audience sizes.

\section{RESULTS AND DISCUSSION}

Frequency rate analysis: The answers from the performance organizer's questionnaire showed that in general, there is a great need for artist safety education and that further in-depth analyses of this aspect are needed.

The frequency analysis showed that the support of the style and the audience and the securing of the viewers were the key items. Most of the respondents were in their $30 \mathrm{sec}$ (as expected) and there was an even response from small, mid-sized and large venues. In all questionnaires, the answers to the following items (as shown in order) were negative:

- "Whether the pre-performance evacuation training guide was a waste of time": 4.58

- "Whether a safety video was watched before a performance": 4.38

- "When a risk was detected whether the staff was notified": 4.09

- "Whether an evacuation route was indicated": 4.4,

- "Whether they checked the safety-related information in the performance brochure": 3.67 .

- A "Definitive No" received a score of 5 as shown in Table 1

\section{Cross-tabulation analysis}

Revenue side: The answers to the questions about the safety education of artists by age group showed that respondents in their $50 \mathrm{sec}$ answered "Yes" while others answered "Usually".

The answers were analyzed to check whether there was a difference in the bypassing of safety rules for accident prevention if it was urgent for a performance

\begin{tabular}{|c|c|c|c|c|c|c|}
\hline Questionnaire item & Definite yes & Yes & Usually yes & No & Definite no & Average \\
\hline \multicolumn{7}{|c|}{ Concert hall safety status } \\
\hline Frequency & 1 & 7 & 18 & 8 & 2 & \multirow[t]{2}{*}{3.08} \\
\hline Ratio & $2.8 \%$ & $19.4 \%$ & $50.0 \%$ & $22.2 \%$ & $5.6 \%$ & \\
\hline \multicolumn{7}{|c|}{ Support from Ministry of Culture, Tourism and Sports } \\
\hline Frequency & 0 & 2 & 22 & 7 & 4 & \multirow[t]{2}{*}{3.37} \\
\hline Ratio & $0.0 \%$ & $5.7 \%$ & $62.9 \%$ & $20.0 \%$ & $11.4 \%$ & \\
\hline \multicolumn{7}{|c|}{ Artist safety training progress } \\
\hline Frequency & 2 & 5 & 10 & 14 & 4 & \multirow[t]{2}{*}{3.37} \\
\hline Ratio & $5.7 \%$ & $14.3 \%$ & $28.6 \%$ & $40.0 \%$ & $11.4 \%$ & \\
\hline \multicolumn{7}{|c|}{ Risk assessment system } \\
\hline Frequency & 0 & 3 & 11 & 15 & 7 & \multirow[t]{2}{*}{3.72} \\
\hline Ratio & $0.0 \%$ & $8.3 \%$ & $30.6 \%$ & $41.7 \%$ & $19.4 \%$ & \\
\hline \multicolumn{7}{|c|}{ Per formance safety ignored } \\
\hline Frequency & 1 & 3 & 10 & 19 & 3 & \multirow[t]{2}{*}{3.56} \\
\hline Ratio & $2.8 \%$ & $8.3 \%$ & $27.8 \%$ & $52.8 \%$ & $8.3 \%$ & \\
\hline
\end{tabular}


according to the age. It was found that respondents in the 20-49 age group answered "Usually" while those in their $50 \mathrm{sec}$ answered "No". In the questions about the provision of reliable support from the Ministry of Culture, Sports and Tourism, the response from all of the occupational groups was "Usually".

Regardless of their age, all answered "Usually". There was no noticeable statistical difference in the perception that emergency evacuation notification and education before the start of a performance was a waste of time by age group.

In the analysis of the experience of viewing safety videos before recent performances according to the age group, all of the respondents answered a "Definite No" regardless of their age. The questionnaire survey was designed to assess whether the respondents had had any experience of watching safety videos before a performance or event.

In the analysis of the experience of speaking to the operating staff when risk factors were found by age group, all respondents answered "No", except those in their $40 \mathrm{sec}$. In the analysis of whether the participants confirmed the route for evacuation of the theater before watching a performance by age group, the respondents answered "No" regardless of their age as shown in Table 2.

\section{Correlation analysis \\ Correlation between safety awareness of performance organizers and safety accident prevention: A correlation was found between questions No. 9, "Do you know the}

risk factors at the venue?" and No. 10, "Did you talk to the staff when you found risk factors at the venue?".

A correlation was found between questions No. 6 , 'Have you ever watched a safety video before a recent performance (event)?" and No. 12, "Have you ever checked the evacuation route for the performance venue before watching a performance?" as shown in Table 3 .

A correlation was found between questions No. 18, "Have you ever blamed a partner after a safety accident?" and No. 24, "Have you received pre-evacuation training and training for your safety?" as shown in Table 4.

Regression analysis: Based on the idea that it is reasonable to estimate the management cost of safety and health at a venue instead of basing it on the number of people or on whether it is an indoor or outdoor space, we derived a model for direct calculation of the safety management rate by substituting the construction amount after applying a practical verification process based on the safety management costs of venues in the actual field.

Table 2: Difference in perception that notification and training for emergency response before the start of a performance are a waste of time per age group

\begin{tabular}{lccccc}
\hline Fields & Frequencies & Average & SD & F & p-value \\
\hline Age 20 29 & 4 & 3.7500 & 0.9574 & 0.08 & 0.987 \\
Age 30 39 & 13 & 3.7692 & 0.8321 & & \\
Age 40 49 & 14 & 3.7857 & 0.8018 & & \\
Age 50 59 & 7 & 3.7143 & 0.7559 & & \\
Over 60 & 7 & 3.5714 & 0.9759 & & \\
\hline
\end{tabular}

Table 3: Correlation analysis of audience questionnaire items

\begin{tabular}{|c|c|c|c|c|c|c|}
\hline Parameters & No. 4 & No. 6 & No. 9 & No. 10 & No. 12 & No. 19 \\
\hline No. 4 Emergency response tips notice before the performance begins & 1 & & & & & \\
\hline No. 6 Safety videos watched before the performance & 0.044 & 1 & & & & \\
\hline \multirow[t]{2}{*}{ No. 9 Risk factors of venue checked } & $\begin{array}{l}0.775 \\
0.038\end{array}$ & -0.057 & 1 & & & \\
\hline & 0.805 & 0.709 & & & & \\
\hline \multirow[t]{2}{*}{ No. 10 Notify staff when a risk factor is found } & 0.109 & 0.014 & $0.438^{* *}$ & 1 & & \\
\hline & 0.477 & 0.926 & 0.003 & & & \\
\hline \multirow[t]{2}{*}{ No. 12 Evacuation route checked } & -0.034 & $0.379^{*}$ & 0.005 & -0.076 & 1 & \\
\hline & 0.824 & 0.010 & 0.973 & 0.622 & & \\
\hline \multirow[t]{2}{*}{ No. 19 Safety-related information in performance brochures checked } & 0.034 & 0.075 & 0.124 & 0.150 & 0.112 & 1 \\
\hline & 0.824 & 0.626 & 0.418 & 0.324 & 0.465 & \\
\hline
\end{tabular}

${ }^{*}$ Correlation coefficient at the 0.05 level (both sides), ${ }^{* *}$ Correlation coefficient at the 0.01 level (both sides)

Table 4: Correlation analysis of organizer questionnaire items (Kim, 2015)

Parameters

No. 3 CEO's safety mindset: high or low

No. 5 Audience safety mindset: high or low

No. 7 Safety organization

No. 9 Artist safety training

No. 18 Whether partner company is blamed after a safety accident

No. 24 Pre-performance evacuation training and education

${ }^{* *}$ Correlation coefficient at the 0.01 level (both sides)

$\begin{array}{rrrrll}\text { No. } 3 & \text { No. } 5 & \text { No. } 7 & \text { No. } 9 & \text { No. 18 } & \text { No. 24 } \\ 1 & & & & & \\ 0.073 & 1 & & & & \\ 0.670 & & & & & \\ 0.290 & 0.105 & 1 & & & \\ 0.086 & 0.541 & & & & \\ 0.008 & 0.044 & -0.078 & 1 & & \\ 0.966 & 0.801 & 0.657 & & & \\ 0.265 & 0.020 & -0.056 & -0.024 & 1 & \\ 0.142 & 0.914 & 0.761 & 0.898 & & \\ -0.110 & -0.070 & 0.006 & -0.198 & 0.453^{* * *} & 1 \\ 0.524 & 0.686 & 0.973 & 0.255 & 0.009 & \\ & & & & & \end{array}$


In addition, extra surveys were conducted by specialists to improve the reliability. Using the data from the questionnaires, the following estimations were made based on the occupational safety and health management cost values for the total construction costs of each building. The statistical analysis process was as follows:

$$
\mathrm{S}_{\mathrm{R}}=\mathrm{A} \cdot \mathrm{M}^{\mathrm{b}}
$$

Where:

$\mathrm{S}_{\mathrm{R}}=$ Performance management cost ratio $(\%)$

$\mathrm{M}$ = Total performance cost (VAT excluded, unit: KRW million)

$\mathrm{A}, \mathrm{b}=$ Constant value

The logarithm for both sides was as follows:

$$
\log \mathrm{S}_{\mathrm{R}}=\log \mathrm{A}+\mathrm{b} \log \mathrm{M}
$$

In Eq. 2 the statistical significant $\mathrm{A}$ and $\mathrm{b}$ could be estimated with a regression analysis (Fig. 1). For example, the process for estimation of the statistically significant

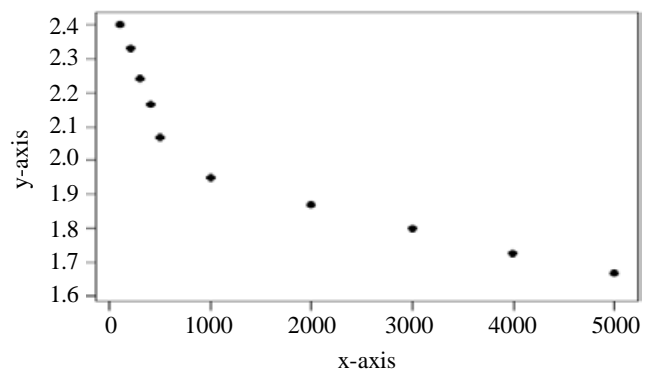

Fig. 1: The proposed estimation equation
$\mathrm{A}$ and $\mathrm{b}$ for the rate calculation at performing arts events was as follows. From the above, it is statistically very significant that the coefficient of determination is very high at $83.1 \%$ and the p-value is $<0.05$. Equation 1 can be obtained by reversing the above regression equation (Fig. 2):

$$
\mathrm{S}_{\mathrm{R}}=1.00531 \mathrm{M}^{-0.3865}
$$

Verification of the equation to calculate safety costs by amount: In the previous study, safety management expenses in the performance industry were introduced and the cost of safety management for performing arts was differentiated according to the audience size. For performances with expected audiences of 1,000 or more, the safety management expenses should be $1.15 \%$ or more; for performances with expected audiences of 3,000 or more, the safety management expenses should be $1.21 \%$ or more. This is illustrated in Table 5-7.

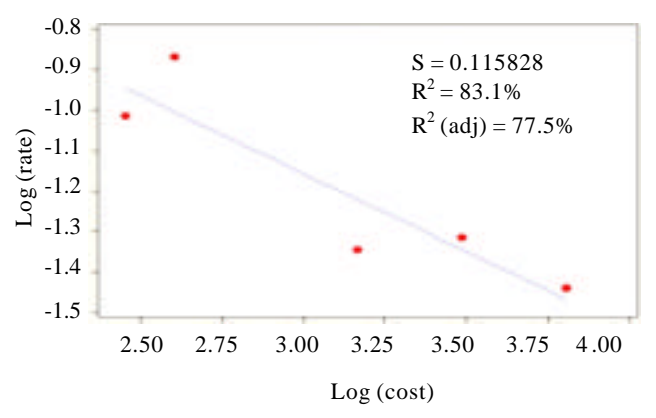

Fig. 2: Concert hall degree of scatter depending on the price of the event (Fitted line plot, Log $($ rate $)=0.0023-0.3865 \log ($ cost $))$

Table 5: Comparison of performance safety management costs

\begin{tabular}{llcll}
\hline Audience No. & Classification & Event cost (million) & Rate & Log (cost) \\
90,000 & Outdoor rock festival & 6453 & 0.0362 & 3.809762 \\
20,000 & Outdoor food festival & 1469 & 0.0450 & -1.44129 \\
500 & Small indoor event & 3066 & 0.0483 & -1.34679 \\
700 & Small indoor event & 283 & 0.0970 & 3.4867022 \\
20,000 & Large-scale outdoor event & 400 & 0.01355 & -1.31605 \\
\hline
\end{tabular}

Table 6: Result of regression analysis for performance safety management fee

\begin{tabular}{llccr}
\hline Audience No. & Classification & Event cost (million) & Actual rate (\%) & Regression rate $(\%) \mathrm{S}_{\mathrm{R}}=1.00531 . \mathrm{M}^{-0.3865}$ \\
\hline 90,000 & Outdoor rock festival & 6453 & 3.6 & 3.4 \\
20,000 & Outdoor food festival & 1469 & 4.5 & \\
500 & Small indoor event & 3066 & 4.8 & \\
700 & Small indoor event & 283 & 9.7 & \\
20,000 & Large-scale outdoor event & 400 & 13.6 & 1.3 \\
\hline
\end{tabular}

Table 7: Comparison of performance safety management expenses

\begin{tabular}{llccc}
\hline Audience No. & Classification & Event cost (million) & Rate & Log (cost) \\
90,000 & Outdoor rock festival & 6453 & 0.0362 & 3.809762 \\
20,000 & Outdoor food festival & 1469 & 0.0450 & 3.167022 \\
500 & Small indoor event & 3066 & 0.0483 & 3.486572 \\
700 & Small indoor event & 283 & 0.0970 & -1.34679 \\
20,000 & Large-scale outdoor event & 400 & -1.31605 \\
\hline
\end{tabular}


However, we found that the difference between the legal safety management costs and the safety management costs spent on actual performances exceeded $10 \%$. For this reason, the above regression analysis equation was proven to be applicable to the calculation of safety management costs using the actual costs spent on performances.

Analysis: We have thus far calculated the rates for each event with statistical analyses, frequency analyses, variance analyses, correlation analyses and regression analyses.

Stage supervisors mainly fell into the 30-39 age group (56.3\%), followed by the $20-29$ age group ( $37.5 \%$ ). This was the highest position occupied by the younger generation (30-39 years old). They were younger than the organizers of the performances by 10 years. This may imply that the safety perspectives of managers and technicians may differ.

While there are directors, sound technicians, lighting technicians and video supervisors among stage supervisors, most of the opinions were from stage supervisors $(62.54 \%)$. Among the stage supervisors, $28.1 \%$ of the respondents worked in small-scale venues, $28.1 \%$ in mid-scale venues and $34.4 \%$ in large-scale venues. This was very similar to the distribution of the organizers.

The age of the audience members who answered the questionnaires was as follows: $28.9 \%$ between 30 and 39 years old, $31.1 \%$ between 40 and 49 years old and $15.6 \%$ between 50 and 59 years old. The lack of enthusiasm of aged participants and the correlation between costs and younger age groups should be further studied.

The occupational composition of the audience members was as follows: $37.8 \%$ were general office workers while $20 \%$ were students. Therefore, it is understood that causal factors such as active interest and cost procurement should be considered.

In terms of audience capacity, $24.4 \%$ was small, $22.2 \%$ was medium, 27.5 was large and $26.7 \%$ involved an outdoor space. It can be assumed that the size of outdoor events such as the Pangyo performance (in which an accident occurred) can be held in the range of $1 / 4$ of the size of the outdoor event.

The factors showing a score higher than 3.5 by subject were analyzed with analyses of variance and correlation analyses. The results of the statistical analyses were further analyzed.

\section{CONCLUSION}

As previously mentioned, safety accidents occur regularly due to failure to get acquainted and comply with the law. Therefore, this survey identified the problems and causes linked to accidents that may occur in venues through an investigation and statistical analysis of previous studies that can be highly applicable to real cases.

Securing safety at performance events requires safety consciousness from all three parties (i.e. the organizer of the performance, the stage supervisor and the spectators). It is also important to promote a safety mindset in the sponsoring party who controls the budget. It is believed that preliminary safety management expenses are important regardless of the performance size, whether small, medium or large.

There is a generation's difference between performance organizers and stage supervision experts. The coordination of opinions and of the authority on safety management cost calculations and preliminary check applications are thought to be just as important as the resetting of the mindset and awareness to secure the venue.

All three safety-related parties (the performance organizers, stage directors and spectators) placed almost equal importance on the size of the performance be it large-scale, medium-scale, small-scale or an outdoor event. This confirms that the same amount of safety assurance expenditure is required for indoor and outdoor events.

\section{ACKNOWLEDGEMENT}

This study was performed with partial funding from a Seoul National University of Science and Technology grant.

\section{REFERENCES}

Choi, J.I., 2016. Making an active cultural industry and event safety. J. Fusion Secur., 16: 58-59.

Kim, S.U., 2015. Maintaining a safe stage at an event hall. Masters Thesis, Seoul National University of Technology, Seoul, South Korea.

Ko, K.H., 2018. Determining efficient safety patrol team operations on construction sites. J. Eng. Appl. Sci., 13: $2732-2736$.

Park, Y.G., 2014. Improving event safety level at a small-size event hall. J. Safety Korea Soc. Safety Eng., 29: 22-27.

Steele, J., 1996. Theatre Building. Academy Editions, London, UK. 